\title{
FRECUENCIA DE DESÓRDENES MENTALES EN ADULTOS MAYORES RESIDENTES EN UNA INSTITUCIÓN DE ASISTENCIA SOCIAL EN LA CIUDAD DE MÉXICO
}

\author{
Jorge Luis López Jiménez1 \\ Guadalupe Barrios Salinas ${ }^{2}$ \\ Blanca Estela López Salgado ${ }^{3}$ \\ María Patricia Martínez Medina ${ }^{4}$ \\ Laura Angélica Bazaldúa Merino 5 \\ Tomás Cortés Solís 6
}

\section{Resumen}

Antecedentes: La Organización Mundial de la Salud ha informado que en los adultos mayores se incrementará y agravará la magnitud de los problemas de salud mental, lo que estará relacionado al incremento de su expectativa de vida y al aumento de las personas que alcanzarán edades avanzadas. Objetivos: Identificar y estimar la frecuencia de desórdenes mentales en adultos mayores. Material y método: Estudio observacional, descriptivo y transversal. Se aplicó un cuestionario que incluía variables sociodemográficas y la Mini Entrevista Neuropsiquiátrica Internacional (M.I.N.I.). Población de estudio de 60 años y más, residentes en una Institución de Asistencia Social de la Ciudad de México. La muestra se seleccionó aleatoria y sistemáticamente, eligiendo una de cada cuatro. Participó personal previamente capacitado tanto en la metodología como en los procedimientos y aplicación del instrumento. Resultados: De la población de estudio, se

\footnotetext{
1 Maestro en Psicología Clínica. Alumno Doctorante en Ciencias Sociales y Humanidades. Área de Psicología Social de Grupos e Instituciones. Universidad Autónoma Metropolitana, Unidad Xochimilco. México, D. F. Investigador de Tiempo Completo. Dirección de Investigaciones Epidemiológicas y Sociales. INP-RF. México D. F. E-mail: jlljimenez@yahoo.com.mx / lopezjl@imp.edu.mx

2 Enfermera Especialista en Atención Primaria a la Salud (EEAPS). Instituto Mexicano del Seguro Social (IMSS). Unidad de Medicina Familiar Número 46. México, D. F. E-mail: jlljimenez@yahoo.com.mx

3 Maestra en Psicología. Consultora en Desarrollo Organizacional. México D. F. E-mail: lopezblancaa@ yahoo.com.mx

4 Psiquiatra. Centro Michoacano de Salud Mental. Secretaria de Salud Michoacán, México. Alumna de la Maestría en Ciencias Médicas, UNAM. México D. F. E-mail: patymtz18@yahoo.com

5 Geriatra. Master en Gerontología Social. Presidenta en Funciones en la Asociación Mexicana de Gerontología y Geriatría. Sistema Nacional para el Desarrollo Integral de la Familia (DIF). Universidad Nacional Autónoma de México, Facultad de Medicina, UNAM. México D. F. E-mail: bamela1@hotmail.com

6 Maestro en Psicología. Profesor e Investigador. Departamento de Educación y Comunicación. Universidad Autónoma Metropolitana, Unidad Xochimilco, México D. F. E-mail: kortestomas@hotmail.com
} 
obtuvo una muestra ponderada de 80 entrevistas completas (67\%). En su mayoría fueron femeninos y viudas, con una media de edad de 80 años y 5 de escolaridad. Encontramos ausencia en el 30\%; en un 40\% sólo se reportó sintomatología y en el 30\% restante se cubrieron diagnósticos psiquiátricos. Conclusiones: La salud mental y los desórdenes psiquiátricos han sido escasamente conocidos y estudiados en esta población. La entrevista MINI permitió identificar y estimar la frecuencia de síntomas y diagnósticos de desórdenes mentales. Nuestra estimación (30\%) fue similar a la reportada en otro estudio (29.7\%). La prevención, identificación, detección e intervención oportuna a los problemas de salud mental adquieren gran importancia en este contexto.

Palabras clave: Adultos mayores. Envejecimiento. Vejez. Desórdenes mentales. Mini Entrevista Neuropsiquiátrica Internacional.

\section{Introducción}

El envejecimiento y la vejez plantean una compleja condición humana y social que conlleva importantes necesidades de investigación, atención a la salud, cuidados y de asistencia social, entre otras. En México, como consecuencia de la transición demográfica y epidemiológica, los adultos mayores conforman un grupo que se incrementa en números absolutos y relativos con mucha rapidez (MÉXICO ..., 2001; HAM CHANDE, 2003). Al respecto, la Organización Mundial de la Salud (OMS) (WORLD HEALTH ..., 1996b) ha indicado que México ocupará el séptimo lugar entre las poblaciones envejecidas más elevadas del mundo en el año 2020.

Diversos informes (WORLD HEALTH .,,, 1996a, 1996b, 2001) señalan que en la población de adultos mayores se incrementarán y agravarán la magnitud de los problemas de salud mental, lo cual estará relacionado con el incremento de la expectativa de vida de las personas con trastornos mentales y con el aumento de los individuos que alcanzarán edades avanzadas, donde el riesgo de presentar desórdenes mentales es elevado. Con el aumento de la esperanza de vida, se han observado mayores tasas de enfermedades mentales; sin embargo, aún cuando la carga asociada a la salud y debida a los trastornos mentales y del comportamiento se incrementan, las estadísticas generadas tienen limitaciones importantes, al no considerar la morbilidad y la discapacidad relacionada (GEZAIRY, 2001). En un reporte reciente, se destaca que en el mundo las personas mayores encabezan las listas de la OMS (WORLD FEDERATION ..., 2007) de nuevos casos de enfermedad mental, 236 por cada 100.000 presentan algún desorden mental. 
Hoy día la noción de salud mental no se restringe a la ausencia de desórdenes mentales, ya que también hay aspectos conductuales y emocionales que pueden afectar positiva o negativamente la calidad de vida de los individuos (GEZAIRY, 2001). El envejecimiento es un proceso en el cual se observa una disminución gradual de la salud física y mental; en el anciano es posible notar diferencias individuales en torno a su salud y enfermedad mental (CRAIG, 1988). Los adultos mayores tienden a presentar por lo común patologías múltiples, por lo que existe la posibilidad de aquellos que sufren trastornos mentales estén afectados también por otra condición médica crónica. Los ancianos frente a esta situación, tienden a buscar tratamiento por sus problemas físicos más que por los mentales, de tal manera se señala que el tratamiento de las alteraciones mentales será más efectivo si está incorporado al sistema de salud general (DESJARLAIS et al., 1997).

Si bien, no existe una enfermedad mental única en los adultos mayores, sí se ha mencionado una amplia gama de ellas, cuya prevalencia tiende a incrementarse con la edad (WORLD HEALTH ..., 1996a, 2001), destacando en este sentido la esquizofrenia, depresión, demencia, trastornos afectivos y de ansiedad; así como otras formas de enfermedades psiquiátricas crónicas, cada una con signos y síntomas característicos. Otros reportes también indican un incremento en la población de adultos mayores y una mayor frecuencia y gravedad de alteraciones en su salud mental (WORLD HEALTH ..., 2001; GEZAIRY, 2001).

Para los trastornos de tipo depresivo (WORLD HEALTH ..., 2001), se ha estimado que entre un $8 \%$ y $20 \%$ de personas mayores en ámbitos comunitarios lo presentan y que en el primer nivel de atención alcanza el $37 \%$. Es de notar que los desórdenes depresivos entre los adultos mayores por lo común no sean detectados, ya que en muchas ocasiones son considerados como parte normal del proceso de envejecimiento. Aún cuando el Alzheimer es el padecimiento más frecuentemente observado en esta población, los adultos mayores son susceptibles de presentar otras alteraciones mentales y del comportamiento. El trastorno es diagnosticado cuando la declinación es suficientemente evidente y afecta las actividades de la vida diaria. El deterioro cognoscitivo se ha relacionado a cambios de la personalidad (suspicacia patológica), humor (depresión o irritabilidad), conducta (descuido de la higiene personal), y orientación espacial (vagar sin rumbo y perderse).

En un reporte (DESJARLAIS et al., 1997), se informó de una prevalencia de trastornos psiquiátricos del 29.7\%; de los cuales el 5.5\% correspondieron a demencias, $14.3 \%$ a depresión, $7.7 \%$ a neurosis, $15.3 \%$ a trastornos de personalidad, $3.3 \%$ por trastornos relacionados con el abuso de sustancias 
y $6.6 \%$ a reacciones de adaptación. En este mismo trabajo, donde se aborda la salud mental en el mundo, se identifican cinco síntomas indicadores de trastorno psiquiátrico: dificultades en el sueño, preocupaciones y ansiedad, pérdida de interés, cansancio y mala memoria.

La enfermedad mental tanto en los países desarrollados como en aquellos en vías de desarrollo constituye causa de sufrimientos, misma que se manifiesta en la desesperanza y aflicción de los individuos, angustia en las familias y pérdidas económicas y sociales, consecuencia de la disminución de la productividad y el aumento en la utilización de los servicios de salud y de asistencia social. Por lo que es necesario reconocer y tratar oportunamente a los adultos mayores que puedan estar presentando alteraciones mentales (DESJARLAIS et al., 1997).

Por lo anteriormente descrito, el interés y propósito del presente trabajo fue el de identificar y estimar la frecuencia de desórdenes mentales en personas de 60 años y más, residentes en una Institución de Asistencia Social (IAP-JAP7) de la Ciudad de México. La información que se presenta forma parte de una línea de investigación (LÓPEZ JIMÉNEZ, 2004; LÓPEZ JIMÉNEZ et al., 2002, 2003, 2005), sobre "Condiciones de vida y salud mental en adultos mayores, Fase 1", que se está llevando a cabo en el Instituto Nacional de Psiquiatría, Ramón de la Fuente (INP-RF).

\section{Material y Métodos}

La investigación llevada a cabo fue de tipo observacional, transversal y descriptiva, con información obtenida mediante la aplicación por entrevista directa de un instrumento en formato de cuestionario, que consta de ocho secciones integradas por el investigador responsable. Para este trabajo se utilizó la información derivada de la sección demográfica y de la Mini Entrevista Neuropsiquiátrica Internacional (M.I.N.I.). Este instrumento es una breve entrevista diagnóstica estructurada desarrollada en Francia y Estados Unidos, la cual explora en los adultos sus principales trastornos psiquiátricos y puede ser aplicada por entrevistadores no especializados. Incluye todos los síntomas del Manual Diagnóstico y Estadístico de los Trastornos Mentales (DSM-IV) y la Clasificación Internacional de Enfermedades (ICD-10), los diagnósticos son establecidos durante la entrevista, las respuestas son dicotómicas y evalúa la presencia de los desórdenes más

7 Institución de Asistencia Privada (IAP), perteneciente a la Junta de Asistencia Privada (JAP). 
comunes; así mismo, confirma la severidad de los síntomas (SHEEHAN et al., 1998; FERRANDO et al., 2000; HEINZE MARTÍN et al., 2000).

Se tomó como población de estudio a los adultos mayores que se encontraban residiendo en la Fundación de Socorros “Agustín González de Cosío”, ubicada al Norte de la Ciudad de México. De esta población se seleccionó una muestra aleatoria sistemática, con base al listado proporcionado por la institución. La fracción de muestreo fue incluyendo a una de cada cuatro personas, también se eligió al azar el número de inicio de la selección.

La aplicación del instrumento se llevó a cabo de acuerdo a la hoja de control elaborada ex profeso, donde se anotaban los datos básicos de la persona elegida. Mismos datos se anotaron en la carátula del cuestionario, a fin de localizar al adulto mayor dentro de la casa hogar, presentarse, aclararle los propósitos del estudio, solicitar su participación, obtener su consentimiento firmado e iniciar la entrevista.

La investigación en su contexto general cubrió las normas éticas, pues no representó riesgo alguno para los entrevistados; además de que no se realizó ninguna estrategia de intervención ni de modificación de variables fisiológicas, psicológicas o sociales. Asimismo, se respetó toda tentativa de rechazo y los datos obtenidos fueron manejados con absoluta confidencialidad y anonimato. Se obtuvo su consentimiento por escrito, aclarando que la información proporcionada sería utilizada únicamente con fines de investigación.

En el desarrollo del trabajo de campo se trató de interferir lo menos posible con las actividades que se desempeñan en la institución. Es de notar que el personal de la casa hogar nos apoyo y brindó las facilidades necesarias en la realización de la fase empírica. Para la aplicación de las entrevistas se integró un equipo de investigación en el cual participó personal de enfermería, psicología, psiquiatría y medicina, con amplia experiencia en el campo de la investigación y aplicación de entrevistas. Previo al trabajo de campo, se brindó capacitación en el manejo de la metodología, procedimientos y en la aplicación del cuestionario por entrevista directa. Para el adiestramiento en la entrevista M.I.N.I., se contó con participación de un médico residente de psiquiatría del INP-RF. La fase empírica del estudio se desarrolló en el mes de diciembre del 2001.

\section{Resultados}

De una población de 117 adultos mayores que se encontraban residiendo en la Casa Hogar al momento de llevar a cabo el trabajo de campo, 
se seleccionó una muestra aleatoria sistemática de 30 adultos. En la parte inicial de los análisis procedimos a asignar a cada miembro de la muestra su peso correspondiente, ya que habíamos entrevistado a uno de cada cuatro $(4 * 30=120)$. El proceso de ponderación consistió en multiplicar a la muestra captada por la fracción de muestreo.

De esta forma, de la muestra ponderada se logró completar el 66.6\% $(n=80)$ de las entrevistas, en el 33.3\% $(n=40)$ restante no se pudieron concluir por problemas de comprensión o no estar disponibles (Figura 1). La información que se presenta corresponde a las entrevistas realizadas. El $65 \%$ de éstas tuvo una duración de entre una y dos horas; el tiempo promedio de aplicación fue de una hora con 54 minutos.

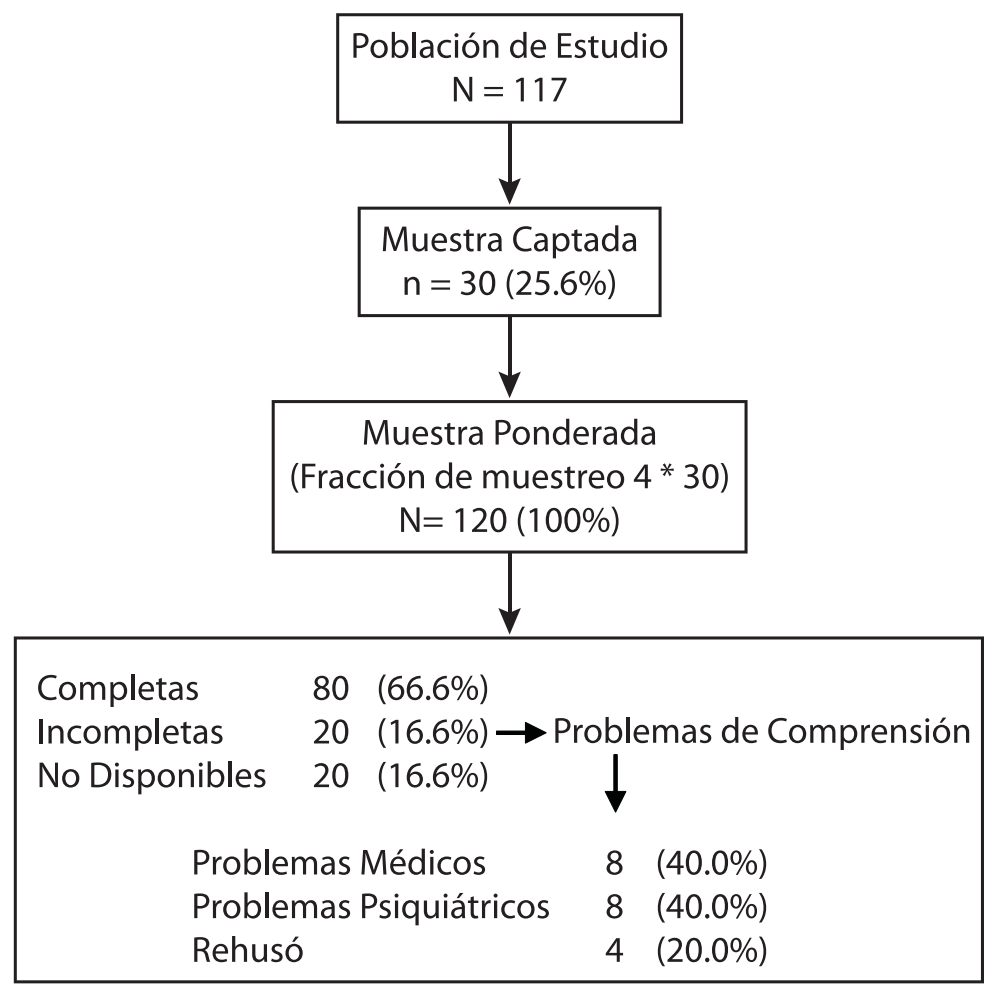

Figura 1 - Distribución de la población de estudio, muestra captada y muestra ponderada.

Con relación a las características demográficas (Tabla 1), sobresale que los mayores porcentajes correspondieran al sexo femenino (65\%), que un 45\% se ubicara entre los 75 y 84 años (media de edad de 80.3, desviación 
estándar de 7.7 y un rango de 64 a 94 años), ser viudo (45\%) y que el 80\% reportara saber leer y escribir (educación no formal); de estos, el 68.7\% obtuvo el nivel primaria de escolaridad (media de 5 años, desviación estándar de 2). Dentro de los principales motivos de estancia a la Casa Hogar encontramos el "No tener quién lo cuidara” (45\%) y los “Problemas de Salud” (30\%), con un tiempo promedio de residencia en la institución de 4.3 años.

Tabla 1 - Características sociodemográficas, por sexo, edad, estado civil, saber leer y escribir y escolaridad $(\mathbf{N}=\mathbf{8 0})$. Ciudad de México, Dic. 2001.

\begin{tabular}{lcc}
\hline & $\mathbf{n}$ & $\mathbf{\%}$ \\
\hline Sexo & 52 & 65.0 \\
$\quad$ Femenino & 28 & 35.0 \\
$\quad$ Masculino & & \\
Edad & 20 & 25.0 \\
$\quad 65$ a 74 & 36 & 45.0 \\
75 a 84 & 24 & 30.0 \\
$\quad 85$ a 98 & & \\
Estado Civil & 20 & 45.0 \\
$\quad$ Viudo & 24 & 30.0 \\
$\quad$ Soltero / Separado & 20 & 25.0 \\
$\quad$ Casado / Unión libre & & \\
Saber leer y escribir & 64 & 80.0 \\
$\quad$ Si & 16 & 20.0 \\
$\quad$ No & & \\
Escolaridad* & \\
$\quad$ Educación no formal & 16 & 25.0 \\
$\quad$ Primaria & 44 & 68.7 \\
$\quad$ Secundaria & 4 & 6.2 \\
\hline Sólo se incluyen a los que informaron saber leer y escribir.
\end{tabular}

De acuerdo a los resultados obtenidos en la aplicación de la Entrevista Diagnóstica de Trastornos Mentales MINI y al ajustar la información a la ausencia/presencia de síntomas y/o diagnósticos de trastornos, encontramos ausencia de estos en el 30\% de las entrevistas; no obstante, en un $40 \%$ se encontró presencia de sintomatología y en el otro 30\% restante, se cubrieron los criterios diagnósticos. 
Destaca en este sentido, el reporte de presencia de trastornos psiquiátricos en el $70 \%$ de la muestra (Tabla 2). Los principales síntomas encontrados fueron los trastornos de angustia, de ansiedad y el antisocial de la personalidad, con porcentajes del $28.5 \%$ en cada uno. En cuanto a los desórdenes psiquiátricos identificados por orden de importancia, corresponden al diagnóstico de Riesgo Suicida (42.8\%) y dentro de estos, el 83.3\% obtuvo el nivel leve y el 16.6\% el alto; seguido del Episodio Depresivo Mayor (14.2\%) y el Trastorno Distímico (7.1\%). Cabe aclarar que, en algunos casos se obtuvieron tres (16.6\%), dos (16.6\%) o sólo un diagnóstico (66.6\%). En cuanto a los que sólo reportaron sintomatología y no alcanzaron a cubrir el criterio diagnóstico; reportaron cuatro (25.0\%), tres (12.5\%), dos (12.5\%) y un síntoma (50.0\%).

Tabla 2 - Resultados Entrevista Psiquiátrica Mini Presencia de Desórdenes Mentales por Síntomas y Diagnósticos ( $=56$ - 70\%). Ciudad de México, Dic. 2001.

\begin{tabular}{|c|c|c|c|c|c|}
\hline & $\mathbf{n}$ & $\%$ & & $\mathbf{n}$ & $\%$ \\
\hline Síntomas & 32 & 40.0 & Diagnósticos & 24 & 30.0 \\
\hline \multirow[t]{2}{*}{ Transtorno de Angustia } & 16 & 28.5 & Episodio Depresivo Mayor & & \\
\hline & & & Sintomas & 20 & 35.7 \\
\hline \multirow{2}{*}{$\begin{array}{l}\text { Estado de Estrés Postrau- } \\
\text { mático }\end{array}$} & 4 & 7.1 & Diagnóstico & 8 & 14.2 \\
\hline & & & Transtorno Distímico & & \\
\hline \multirow[t]{2}{*}{ Transtornos Psicóticos } & 4 & 7.1 & Sintomas & 32 & 57.1 \\
\hline & & & Diagnóstico & 4 & 7.1 \\
\hline \multirow{2}{*}{$\begin{array}{l}\text { Transtorno de Ansiedad } \\
\text { Generalizada }\end{array}$} & 16 & 28.5 & Riesgo Suicida & & \\
\hline & & & Diagnóstico & 24 & 42.8 \\
\hline \multirow{2}{*}{$\begin{array}{l}\text { Transtorno Antisocial de la } \\
\text { Personalidad }\end{array}$} & 16 & 28.5 & Leve & 20 & 83.3 \\
\hline & & & Alto & 4 & 16.6 \\
\hline
\end{tabular}

Finalmente como se muestra en el Tabla 3, de acuerdo a sus características demográficas y su distribución por reporte de sintomatología, obtención de un diagnóstico y/o ambos en la muestra ponderada estudiada, observamos que, con relación al género, en las tres situaciones predominó el sexo femenino, por edad en el rango de 85 a 96 años se ubicó el mayor porcentaje para síntomas, en cambio en los 75 a 84 años el $66.6 \%$ cubrió el criterio diagnóstico, para el total estos dos rangos de edad se obtuvo el mismo porcentaje (35.7\%). Por estado civil, el mayor porcentaje de reporte de síntomas fue para soltero/viudo, en el caso de los que obtuvieron diagnóstico y el total de la muestra, los mayores porcentajes fueron para ser viudo 
(66.6\% y 42.8\% en cada caso). En las tres situaciones el nivel de escolaridad primaria obtiene los mayores porcentajes $60.0 \%$, 66.6\% y 50.0\% respectivamente. Con relación al motivo de estancia, destaca que en el grupo de las personas mayores que sólo reportaron síntomas el "no tener quién lo cuide" y los "problemas de salud" alcanzaran los mayores porcentajes (35.7\% en ambos casos); tanto en el grupo de los que obtuvieron diagnóstico (66.6\%) como en el total de la muestra (50.0\%) el "no tener quién lo cuidara" se encontró el mayor porcentaje.

Tabla 3 - Características demográficas: sexo, edad, estado civil, escolaridad y motivo de estancia por Síntomas y Diagnósticos. Entrevista Psiquiátrica Mini ( $=56$ - 70\%). Ciudad de México, Dic. 2001.

\begin{tabular}{|c|c|c|c|c|c|c|}
\hline & \multicolumn{2}{|c|}{$\begin{array}{l}\text { Síntomas } \\
(\mathrm{n}=32)\end{array}$} & \multicolumn{2}{|c|}{$\begin{array}{c}\text { Diagnósticos } \\
(\mathrm{n}=24)\end{array}$} & \multicolumn{2}{|c|}{$\begin{array}{c}\text { Total } \\
(N=56)\end{array}$} \\
\hline & $\mathbf{n}$ & $\%$ & $\mathbf{n}$ & $\%$ & $\mathbf{N}$ & $\%$ \\
\hline \multicolumn{7}{|l|}{ Sexo } \\
\hline Masculino & 12 & 37.5 & 4 & 16.6 & 16 & 28.5 \\
\hline Femenino & 20 & 62.5 & 20 & 83.3 & 40 & 71.4 \\
\hline \multicolumn{7}{|l|}{ Edad } \\
\hline 64 a 74 & 12 & 37.5 & 4 & 16.6 & 16 & 28.5 \\
\hline 75 a 84 & 4 & 12.5 & 16 & 66.6 & 20 & 35.7 \\
\hline 85 a 96 & 16 & 50.0 & 4 & 16.6 & 20 & 35.7 \\
\hline \multicolumn{7}{|l|}{ Estado Civil } \\
\hline Viudo & 8 & 25.0 & 16 & 66.6 & 24 & 42.8 \\
\hline Soltero / Separado & 20 & 62.5 & - & - & 20 & 35.7 \\
\hline Casado / Unión Libre & 4 & 12.5 & 8 & 33.3 & 12 & 21.4 \\
\hline \multicolumn{7}{|l|}{ Escolaridad* } \\
\hline Primaria & 12 & 60.0 & 20 & 83.3 & 32 & 72.7 \\
\hline Secundaria y Profesional & 4 & 20.0 & - & - & 4 & 9.0 \\
\hline Leer y Escribir & 4 & 20.0 & 4 & 16.6 & 8 & 18.1 \\
\hline \multicolumn{7}{|l|}{ Motivo de Estancia } \\
\hline No tener quien lo cuide & 12 & 37.5 & 16 & 66.6 & 28 & 50.0 \\
\hline Problemas de Salud & 12 & 37.5 & 8 & 33.3 & 20 & 35.7 \\
\hline Sin Recursos Económicos & 8 & 25.0 & - & - & 8 & 14.2 \\
\hline
\end{tabular}

* Sólo se incluyen a los que informaron saber leer y escribir. 


\section{Discusión}

Los adultos mayores constituyen un grupo vulnerable dentro de la sociedad. El estado de salud mental y los desórdenes psiquiátricos han sido los menos conocidos y estudiados en este grupo hasta el momento.

Con respecto a la Mini International Neuropsychiatric Interview (M.I.N.I.), probó ser útil para detectar tanto sintomatología, como en el establecimiento de los criterios diagnósticos de los módulos investigados en el instrumento.

En la literatura se han reportado algunas alteraciones mentales prevalentes, como el caso de las demencias, la depresión y el deterioro cognoscitivo; no obstante, existe una amplia gama de padecimientos mentales que es necesario detectar, identificar y diagnosticar, para poder brindar la atención requerida de manera oportuna.

La prevalencia diagnóstica de desordenes psiquiátricos reportados en nuestra investigación (30\%) fue similar a la descrita por la Organización Panamericana de la Salud (OPS) (DESJARLAIS et al., 1997) en un informe (29.7\%). Sin embargo, en nuestro estudio se logró establecer que un $40 \%$ de la muestra cursaba con sintomatología de naturaleza psiquiátrica, sobresaliendo los trastornos de angustia, de ansiedad y el antisocial de la personalidad. Los criterios diagnósticos encontrados son consistentes a lo informado en la literatura: riesgo suicida, episodios depresivos mayores y trastorno distímico. Es importante aclarar que, en el caso del riesgo suicida (leve), el reactivo por el cual cubrieron el diagnóstico fue por: ¿Haber pensado que estaría mejor muerto o ha deseado estar muerto?, mismo que investiga "ideación de muerte” mas que "ideación suicida”.

Con relación a los 16 módulos que explora el M.I.N.I., sólo se encontró sintomatología en siete de ellos y únicamente en tres se cubrieron los criterios diagnósticos. También es de notar haber observado diferencias en la distribución por edad, estado civil y en una categoría del motivo de estancia de acuerdo al reporte de síntomas, los criterios diagnósticos y en ambas situaciones.

Para futuras investigaciones se plantea la necesidad de aumentar los tamaños de muestra y la aplicación en otros ámbitos al realizado en el presente estudio, esto con la finalidad de estimar la confiabilidad y validez de la entrevista M.I.N.I.; así como su funcionamiento y adaptación a otros contextos.

Finalmente, es necesario destacar la importancia de la detección y diagnóstico oportunos de los problemas de salud mental en los adultos ma- 
yores, con el propósito de poder brindar la atención requerida especializada a este grupo de nuestra población.

\title{
MENTAL DISORDERS FREQUENCY IN ELDERLY LIVING IN A SOCIAL ASSISTANCE INSTITUTION IN MEXICO CITY
}

\begin{abstract}
Background: World Health Organization has reported that in old adults population will increase and aggravate the magnitude of mental health problems, which will be linked to the increase in his life expectancy and with the rise of individuals who reach old age. Objectives: identify and estimate the frequency of mental disorders in elderly people. Materials and methods: Study observational, descriptive and transversal. It applies a questionnaire, which included socio-demographic variables and the Mini International Neuropsychiatric Interview (M.I.N.I.). Study population of persons aged 60 and over living in an Institution of Social Assistance in Mexico City, here were selected a systematic random sample, choosing one in four adults. The interviews were available staff previously trained in the handling of the methodology, procedures and application of the instrument Results: Out of a population study, resulting in a weighted sample of 80 completed interviews (67\%). The main percentages were females and widower, with a mean age of 80 years and 5 of schooling. We find absence in the $30 \%$, only $40 \%$ reported symptoms and the remaining 30\% were filled psychiatric diagnoses. Conclusions: Mental health and psychiatric disorders has been scantily known and studied until this moment. The interview M.I.N.I. allowed us to identify and estimate their frequency of symptoms and diagnostics mental disorders. The frequency diagnostic of mental disorders found in our research (30\%) is very similar to that obtained in another study (29.7\%). In this context, the prevention, detection and opportune intervention for mental health problems are remarkable.

Keywords: Elderly. Aging. Oldness. Mental disorders. Mini International Neuropsychiatric Interview.
\end{abstract}




\section{REFERENCIAS}

CRAIG, Grace J. et al. Desarrollo Psicológico. México, D.F.: Prentice-Hall, 1988.

DESJARLAIS, Robert et al. Salud Mental en el Mundo: problemas y prioridades en las poblaciones de bajos ingresos. Washington, D.C.: Organización Panamericana de la Salud, 1997.

FERRANDO, Laura et al. Mini International Neuropsychiatric Interview (M.I.N.I.): versión en español 5.0.0 DSM-IV. Madrid: Instituto IAP; Tampa: University of South Florida, 2000.

GEZAIRY, H. A. World Health Day 2001 Documents: foreword. Cairo: Eastern Mediterranean Region Office, World Health Organization, 2001. Disponible en: <http://www.emro.who.int/mnh/whd/WHD2001DocumentsForeword.htm>. Acceso en: 27 nov. 2007.

HAM CHANDE, Roberto. El Envejecimiento en México: el siguiente reto de la transición demográfica. Tijauna: El Colegio de la Frontera Norte, 2003.

HEINZE MARTÍN, Gerhard et al. Mini International Neuropsychiatric Interview (M.I.N.I.): Spanish version (South and Central America) 5.0.0 DSM-IV. México, D.F.: Instituto Nacional de Psiquiatría, 2000.

LÓPEZ JIMÉNEZ, Jorge Luis. Estudio de Condiciones de Vida y Salud Mental en Adultos Mayores, Fase I: reporte interno. México, D.F.: Dirección de Investigaciones Epidemiológicas y Psicosociales, Instituto Nacional de Psiquiatría Ramón de la Fuente, 2004.

LÓPEZ JIMÉNEZ, Jorge Luis et al. Condiciones de Salud en Adultos Mayores Institucionalizados: su impacto en el bienestar social. In: CONGRESO AL ENCUENTRO DE LA PSICOLOGÍA MEXICANA, 6.; CONGRESO LATINOAMERICANO DE ALTERNATIVAS EN PSICOLOGÍA, 2002, Puebla. Anales . . México, D.F.: Sociedad Mexicana de Psicología Social, 2002. P. 1-17.

LÓPEZ JIMÉNEZ, Jorge Luis et al. Psychiatric Disorders in Elderly Living in a Social Assistance in Mexico City. In: WORLD CONGRESS OF 
GERONTOLOGY, 18., 2005, Rio de Janeiro. Abstracts . . . Rio de Janeiro: International Association of Gerontology, 2005. P. 614.

LÓPEZ JIMÉNEZ, Jorge Luis et al. Reflexiones Metodológicas de Investigación Psicosocial en Viejos: resultados de un estudio. Archivo Geriátrico, México, D.F., v. 6, n. 3, p. 74-77, 2003.

MEXICO. Consejo Nacional de Población. La Población de México en el Nuevo Siglo. 2. ed. México, D.F., 2001.

SHEEHAN, David et al. The Mini-International Neuropsychiatric Interview (M.I.N.I.): the development and validation of a structured diagnostic psychiatric interview for DSM-IV and ICD-10. Journal of Clinical Psychiatry, Memphis, v. 59, p. 22-33, 1998. Supplement n. 20.

WORLD FEDERATION FOR MENTAL HEALTH. World Mental Health Day: mental health in a changing world: the impact of culture and diversity. Springfield, 2007. Disponible en: <http://www.wfmh.org/PDF/SpanishVersion2007.pdf >. Acceso en: 27 nov. 2007.

WORLD HEALTH ORGANIZATION. Mental Health and Demographic Factors. Geneva, 1996a. (Fact Sheets, 131).

WORLD HEALTH ORGANIZATION. Population Ageing: a public health challenge. Geneva, 1996b. (Fact Sheets, 135).

WORLD HEALTH ORGANIZATION. The World Health Report 2001: mental health: new understanding, new hope. Geneva, 2001.

Recebido em: 16-11-2007

$1^{a}$ revisão: 10-01-2008

$2^{a}$ revisão: $30-06-2008$

Aceite final: 03-07-2008 